\title{
REMOÇÃO DE ÍONS DE COBRE E ZINCO EM SOLUÇÃO AQUOSA USANDO MACROALGA MARINHA PELVETIA CANALICULATA COMO BIOSSORVENTE
}

\author{
F. GIRARDI ${ }^{1}$, F. V. HACKBARTH ${ }^{1}$, R. A. R. BOAVENTURA ${ }^{2}$, V. J. P. VILAR ${ }^{2}$, S. M. A. \\ GUELLI de SOUZA ${ }^{1}$, A. A. ULSON de SOUZA ${ }^{1}$ \\ ${ }^{1}$ Universidade Federal de Santa Catarina, Departamento de Engenharia Química e Engenharia de \\ Alimentos \\ ${ }^{2}$ Faculdade de Engenharia da Universidade do Porto, Departamento de Engenharia Química \\ E-mail para contato: francigirardi@yahoo.com.br
}

\begin{abstract}
RESUMO - A capacidade natural de troca catiônica da macroalga Pelvetia canaliculata foi investigada para a remoção dos metais $\mathrm{Cu}^{2+}$ e $\mathrm{Zn}^{2+}$ de soluções aquosas. Através das técnicas de FTIR e de titulação potenciométrica foi possível determinar que os principais grupos funcionais presentes na superfície da alga responsáveis pela ligação dos metais são os grupos sulfônicos e carboxílicos, representando no total $2,5 \mathrm{mEq} / \mathrm{g}$. Os ensaios de esterificação da biomassa permitiram a quantificação dos grupos sulfônicos $(1,0 \mathrm{mEq} / \mathrm{g}) \mathrm{e}$ grupos carboxílicos $(1,5 \mathrm{mEq} / \mathrm{g})$. Os coeficientes de seletividade de cobre ou zinco vs hidrogênio foram muito menores do que com o sódio, indicando que os íons de hidrogênios podem competir pelos mesmos sítios de ligação, diminuindo fortemente a remoção dos íons de cobre e zinco em baixos valores de $\mathrm{pH}$. A pH 4,0 quase todos os sítios ativos presentes na superfície da alga foram ocupados pelos íons cobre ou zinco $(\sim 2,4 \mathrm{mEq} / \mathrm{g})$.
\end{abstract}

\section{INTRODUÇÃ̃O}

O meio mais usual de contaminação por íons metálicos é através da descarga de efluentes não tratados em ambientes aquáticos. A liberação desses compostos deve-se em grande parte pelas indústrias de mineração, fundição, processamento de minérios, fabricação de equipamentos elétricos, têxtil, couro, baterias, fertilizantes, pesticidas, entre outras (Volesky, 2001). Os íons de cobre e zinco estão entre os metais pesados mais comuns presentes nestes tipos de águas residuárias.

Tendo em vista a importância do controle da poluição da água, um grande número de tecnologias tem vindo a ser desenvolvidas para remover metais tóxicos dos efluentes industriais. Entre essas tecnologias, a biossorção vem ganhando destaque nos últimos anos, pois apresenta como vantagem a utilização de biomassa existente em quantidades abundantes, como por exemplo, algas ou resíduos de outras operações industriais (Volesky, 2001).

A elevada capacidade de remoção de íons metálicos por algas marinhas é devido à presença de vários grupos funcionais na superfície das algas, tais como grupos carboxílicos e sulfônicos. Entre os diferentes tipos de algas, as algas marrons tem provado ser um ótimo biossorvente para remoção de 


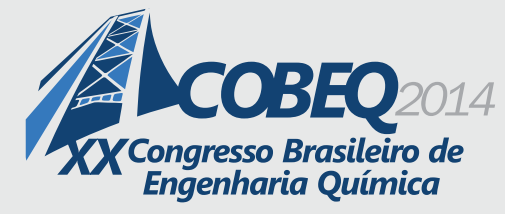

19 a 22 de outubro de 2014

Florianópolis/SC

metais pesados, apresentando muitas vezes capacidades de adsorção semelhantes às resinas de troca iônica. Sendo assim, as algas marinhas podem competir em termos de preço e eficiência com as resinas de troca iônica disponíveis no mercado (Girardi et al., 2014; Hackbarth et al., 2014).

O grande desafio deste trabalho foi avaliar as propriedades de troca catiônica da macroalga marinha marrom $P$. canaliculata para os íons de cobre e zinco, dois dos principais íons encontrados nos efluentes da indústria de processamento de metais. A capacidade de troca iônica, coeficientes de seletividade para a biomassa saturada com íons sódio, como também os coeficientes de difusão intrapartícular, foram avaliados considerando os sistemas ternários $\mathrm{Na}^{+} / \mathrm{H}^{+} / \mathrm{Cu}^{2+}$ e $\mathrm{Na}^{+} / \mathrm{H}^{+} / \mathrm{Zn}^{2+}$, os quais são importantes parâmetros de projeto para um processo de tratamento por troca iônica. Técnicas de FTIR e de titulação potenciométrica, juntamente com a informação fornecida pela esterificação dos grupos funcionais, foram utilizados para a identificação e quantificação dos grupos de ligação ácidos responsáveis pela biossorção de cobre e zinco.

\section{MATERIAIS E MÉTODOS}

\subsection{Reagentes}

Soluções de $\mathrm{Cu}^{2+}$ e $\mathrm{Zn}^{2+}$ foram preparadas a partir da dissolução dos seus respectivos sais $\mathrm{CuCl}_{2} \cdot 2 \mathrm{H}_{2} \mathrm{O}$ (Merck > 98\%) e $\mathrm{ZnCl}_{2}$ (Merck > 98\%) em água ultrapura. A solução 0,5 $\mathrm{M}$ de $\mathrm{NaCl}$ foi preparada por dissolução de cloreto de sódio (Merck, com pureza > 99,5\%) em água deionizada (DI).

\subsection{Preparação da biomassa}

A macroalga marinha marrom $P$. canaliculata foi utilizada neste estudo. A preparação do material foi apresentada num trabalho anterior (Girardi et al., 2014; Hackbarth et al., 2014).

\subsection{Caracterização da biomassa}

A identificação dos grupos funcionais na superfície do biossorvente foi realizada por espectroscopia de infravermelho utilizando o equipamento FTIR IRAffinity (Shimadzu).

A esterificação dos grupos carboxílicos foi realizada suspendendo $2 \mathrm{~g}$ de biomassa em metanol $(130 \mathrm{~mL})$ e $\mathrm{HCl}$ concentrado $(1,2 \mathrm{~mL})$ e deixada em repouso durante $6 \mathrm{~h}$ a $25^{\circ} \mathrm{C}$. Em seguida, a biomassa foi lavada com água deionizada e seca a $45^{\circ} \mathrm{C}$ durante $24 \mathrm{~h}$. Para a esterificação dos grupos sulfônicos, $2 \mathrm{~g}$ de biomassa foi suspensa em $130 \mathrm{~mL}$ de metanol e 1,2 $\mathrm{mL}$ de $\mathrm{HCl}$ concentrado e foi deixada em repouso durante quatro ciclos de $48 \mathrm{~h}$, com agitação contínua, com a substituição da solução metanol/ $\mathrm{HCl}$ entre os ciclos.

\subsection{Estudo cinético e do equilíbrio de troca catiônica}

Os experimentos cinéticos de troca catiônica foram realizados em frascos de vidro de $100 \mathrm{~mL}$ fechados contendo $50 \mathrm{~mL}$ de soluções de $\mathrm{Cu}^{2+}$ ou $\mathrm{Zn}^{2+}(\approx 100 \mathrm{mg} / \mathrm{L})$ e $0,05 \mathrm{~g}$ de alga bruta ou alga-Na $(1 \mathrm{~g} / \mathrm{L})$. Os frascos foram colocados dentro de um refrigerador termostático (Lovibond), a fim de 


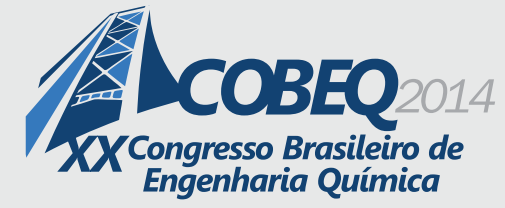

19 a 22 de outubro de 2014

Florianópolis/SC

garantir as condições de temperatura controlada $\left(25^{\circ} \mathrm{C}\right)$. A suspensão foi mantida sob agitação orbital até atingir o equilíbrio $(24 \mathrm{~h})$. As cinéticas de troca catiônica de cobre e zinco foram realizadas em três diferentes valores de $\mathrm{pH}$ inicial $(2,0,3,0$ e 4,0). As amostras foram recolhidas (cada erlenmeyer corresponde a uma amostra) em intervalos de tempo definidos.

Os experimentos de equilíbrio de troca catiônica foram realizados em frascos de vidro de $100 \mathrm{~mL}$ fechados contendo $50 \mathrm{~mL}$ de soluções de $\mathrm{Cu}^{2+}$ ou $\mathrm{Zn}^{2+}$ e $0,05 \mathrm{~g}$ de alga-Na $(1 \mathrm{~g} / \mathrm{L})$. Os experimentos foram realizados em duplicata com as concentrações iniciais de cobre ou de zinco na faixa de 40-360 $\mathrm{mg} / \mathrm{L}$, em três diferentes valores de $\mathrm{pH}$ inicial $(2,0,3,0$ e 4,0).

As amostras (da cinética e do equilíbrio) foram filtradas por filtros de membrana de acetato de celulose (Sartorius Stedim, $45 \mu \mathrm{m}$ de porosidade) e as concentrações de metal residual (metal contaminante $(\mathrm{Cu}$ ou $\mathrm{Zn}))$ e de metais permutáveis $(\mathrm{Ca}, \mathrm{Mg}, \mathrm{Na}$ e/ou $\mathrm{K})$ ) foram determinadas por Espectrofotometria de Absorção Atómica - EAA (GBC 932 Plus).

\subsection{Procedimento analítico}

As concentrações de $\mathrm{Cu}, \mathrm{Zn}, \mathrm{Na}, \mathrm{K}, \mathrm{Ca}$ e $\mathrm{Mg}$ nas soluções aquosas foram determinadas por EAA (GBC 932 Plus) utilizando a correção de Deutério e uma fenda espectral de largura 0,5 nm para Cu, Zn, $\mathrm{Ca}, \mathrm{Na}$ e $\mathrm{K}$, e $1,0 \mathrm{~nm}$ para o $\mathrm{Mg}$. Para a análise de $\mathrm{Ca}$ e $\mathrm{Mg}$, solução de $\mathrm{La}_{2} \mathrm{O}_{3}(5,86 \%$ (m/v)) foi adicionada à solução a analisar $\left(1 \mathrm{~mL} \mathrm{La} \mathrm{O}_{3}\right.$ para $10 \mathrm{~mL}$ de solução a analisar), a fim de minimizar interferências químicas, enquanto que na análise de $\mathrm{Na}$ e $\mathrm{K}, \mathrm{KCl}(0,38 \%$ (m/v)) e $\mathrm{NaCl}(0,50 \%$ (m/v)) foram adicionados, respectivamente, para minimizar as interferências de ionização.

\section{RESULTADOS E DISCUSSÃO}

\subsection{Análises de FTIR}

O espectro de FTIR obtido para a biomassa $P$. Canaliculata bruta ou saturada com íons de sódio, cobre ou zinco, conforme mostra a Figura 1, apresentaram diferentes picos de absorção, sendo possível obter informações sobre as ligações químicas presentes na superfície da biomassa, e correlacionar com os grupos funcionais responsáveis pela ligação dos íons metálicos.

A região entre 3600 e $3200 \mathrm{~cm}^{-1}$ corresponde a bandas de grupos hidroxila, e alongamento $\mathrm{N}-\mathrm{H}$ (aminas e amidas em ácidos aminados). Esta banda é larga e forte (Figura 1) por causa do elevado número e grande densidade de ligações de hidrogênio, principalmente hidroxilas nos anéis polissacarídeos da piranose (Oliveira et al., 2014). O espectro de FTIR mostra um pequeno deslocamento da banda, o que pode ser correlacionado com a interação de íons de cobre e zinco com os grupos $-\mathrm{NH}_{2}$ (Subbaiah et al., 2011). 


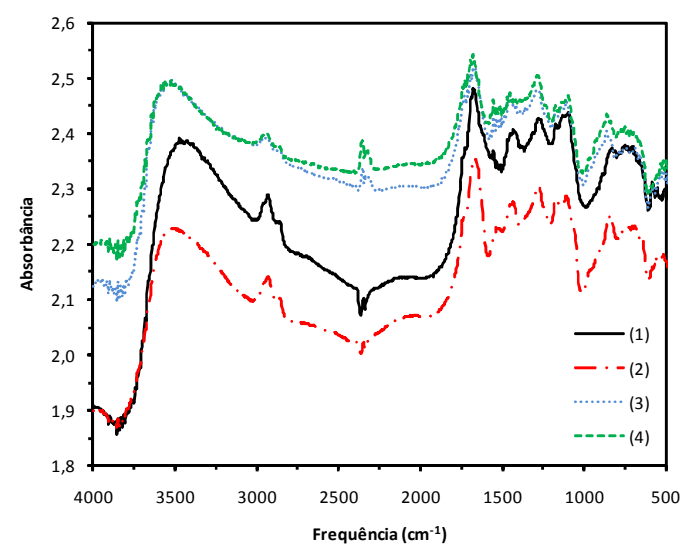

Figura 1 - Espectros de FTIR para diferentes formas da alga: (1) alga bruta, (2) alga-Na, (3) alga-Na após biossorção de cobre, (4) alga-Na após biossorção de zinco.

$\mathrm{O}$ alongamento carbonila dos ácidos carboxílicos aparece próximo a $1750 \mathrm{~cm}^{-1}$ (Oliveira et al., 2014). Uma banda a $1420 \mathrm{~cm}^{-1}$ pode ser devido à vibração de deformação de $\mathrm{C}-\mathrm{OH}$ com a contribuição do estiramento simétrico vibracional O-C-O do grupo carboxilato (Mathlouthi; Koenig, 1987). Para a biomassa carregada com íons de cobre e zinco, observou-se uma mudança destes picos, indicando a interação dos íons metálicos com os grupos carboxílicos. A capacidade de adsorção das algas está diretamente relacionada com a presença destes grupos no polímero de alginato (Davis et al., 2003). As bandas $1260 \mathrm{~cm}^{-1}$ e $1160 \mathrm{~cm}^{-1}$ podem ser atribuídas à presença de grupos éster sulfato $(\mathrm{S}=\mathrm{O})$, que é um componente característico do fucoidan (Gómez-Ordóñez; Rupérez, 2011).

\subsection{Bloqueio dos grupos carboxílicos e sulfônicos presentes na superfície da biomassa}

Os grupos carboxílicos e sulfônicos foram bloqueados tentando elucidar o papel dos grupos funcionais presentes na superfície da alga Pelvetia canaliculata na ligação de $\mathrm{Cu}^{2+} \mathrm{e} \mathrm{Zn}^{2+}$. A capacidade de adsorção obtida no equilíbrio para as algas esterificados foi de $43 \% / 18 \%\left(\mathrm{Cu}^{2+}\right)$ e $55 \% / 58 \%\left(\mathrm{Zn}^{2+}\right)$ menor em comparação com a alga não esterificada (alga-Na) para pH 4,0 e 2,0, respectivamente, o que indica que os grupos carboxílicos desempenham um papel importante na biossorção de $\mathrm{Cu}^{2+} \mathrm{e} \mathrm{Zn}^{2+}$.

Embora o procedimento de esterificação das algas reduzisse substancialmente a capacidade de biossorção dos metais, outros grupos funcionais presentes na superfície da biomassa não foram bloqueados e são responsáveis pelo restante de metal retido na biomassa, como por exemplo, os grupos sulfônicos, os quais estão presentes nos polissacarídeos sulfatados do fucoidan (Davis et al., 2003). Com o bloqueio dos grupos sulfônicos e carboxílicos, a capacidade de biossorção diminuiu 68\%/32\% para $\mathrm{Cu}^{2+}$ e 78\%/62\% para o $\mathrm{Zn}^{2+}$, em pH 4,0/2,0, respectivamente. De acordo com estes resultados, o procedimento de bloqueio não foi capaz de impedir completamente a capacidade de biossorção da resina natural, principalmente para valores de $\mathrm{pH}$ mais baixos, o que indica que os grupos sulfônicos não foram completamente bloqueados usando o procedimento adotado. 


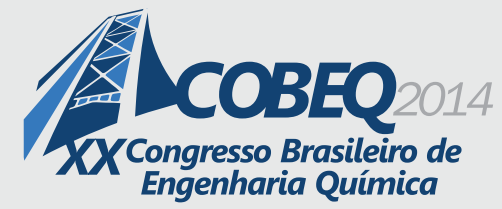

Em nosso estudo anterior (Hackbarth et al., 2014), utilizando técnicas de titulação potenciométrica, constatou-se a presença de dois grandes grupos funcionais (grupos funcionais ácidos e básicos) na superfície da biomassa $P$. canaliculata. Considerando os resultados obtidos na esterificação e na titulação potenciométrica, e os dados relatados em trabalho anterior (Hackbarth et al., 2014), a quantidade de grupos sulfônicos e carboxílicos pode ser assumida como $\sim 1,0 \mathrm{mmol} / \mathrm{g}$ e $\sim 1,5$ $\mathrm{mmol} / \mathrm{g}$, respectivamente, que está de acordo com a quantidade total de íons de sódio presente na superfície da alga-Na $(\sim 2,45 \mathrm{mEq} / \mathrm{g})$ e quantidade total de metais leves na alga bruta $(\sim 2,47 \mathrm{mEq} / \mathrm{g})$.

\subsection{Cinéticas de troca catiônica com alga bruta}

A biomassa bruta foi inicialmente utilizada para realizar a cinética de troca catiônica utilizando uma concentração inicial de metal de $100 \mathrm{mg} / \mathrm{L}$ a pH 4,0, a fim de avaliar o mecanismo de remoção de

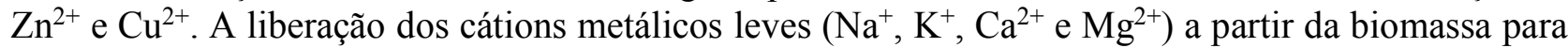
a solução, bem como a adsorção de $\mathrm{Cu}^{2+}, \mathrm{Zn}^{2+}$ e $\mathrm{H}^{+}$pela biomassa foi monitorada.

Verificou-se que a quantidade de $\mathrm{Zn}^{2+}$ e $\mathrm{Cu}^{2+}$ ligado à biomassa bruta $P$. canaliculata foi semelhante a soma de metais leves liberados $\left(\mathrm{Na}^{+}, \mathrm{K}^{+}, \mathrm{Ca}^{2+} \mathrm{e} \mathrm{Mg}^{2+}\right)$, a partir da biomassa para a solução. De acordo com o pH da solução, a adsorção de prótons era negligenciável. Hackbarth et al. (2014) apresentaram valores de concentrações de metais leves de $0,72 \mathrm{mEq} \mathrm{Mg}{ }^{2+} / \mathrm{g}, 0,65 \mathrm{mEq} \mathrm{Na} / \mathrm{g}, 0,56$ $\mathrm{mEq} \mathrm{Ca}{ }^{2+} / \mathrm{g}$ e $0,54 \mathrm{mEq} \mathrm{K} / \mathrm{g}$, para a mesma alga, recolhidas no mesmo local e temporada. De acordo com a cinética de troca catiônica, os íons de potássio e sódio são liberados mais rapidamente e em maiores quantidades do que os íons de magnésio e cálcio, o que indica que a troca de $\mathrm{Cu}^{2+}$ e $\mathrm{Zn}^{2+}$ é

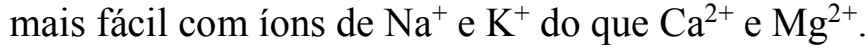

\subsection{Equilíbrio de troca catiônica com alga saturada com sódio}

Coeficientes de seletividade para a alga saturada com íons sódio foram determinados para $\mathrm{Cu}^{2+}$, $\mathrm{Zn}^{2+} \mathrm{e} \mathrm{H}^{+}$, utilizando estudos de equilíbrio ternários em diferentes valores de $\mathrm{pH}$ e concentrações de metais $(\mathrm{Cu}$ e $\mathrm{Zn})$. A lei de ação das massas, considerando os grupos funcionais carboxílicos monovalentes e os grupos sulfônicos inicialmente saturados com íons de sódio, e as reações de troca com $\mathrm{Cu}^{2+}, \mathrm{Zn}^{2+} \mathrm{e} \mathrm{H}^{+}$, foi utilizada para ajustar os dados experimentais para ambos os sistemas ternários, $\mathrm{Na}^{+} / \mathrm{H}^{+} / \mathrm{Cu}^{2+}$ ou de $\mathrm{Na}^{+} / \mathrm{H}^{+} / \mathrm{Zn}^{2+}$. Uma descrição detalhada da equação da lei de ação das massas pode ser encontrada em Hackbarth et al. (2014).

A lei de ação das massas para as misturas ternárias $\left(\mathrm{Na}^{+} / \mathrm{H}^{+} / \mathrm{Cu}^{2+}\right.$ e $\left.\mathrm{Na}^{+} / \mathrm{H}^{+} / \mathrm{Zn}^{2+}\right)$ foi capaz de prever os dados de equilíbrio (Figura 2), com coeficientes de seletividade $\alpha_{N a}^{C u}=521, \alpha_{N a}^{Z n}=341$ e $\alpha_{N a}^{H}=10$ para os grupos carboxílicos e $\alpha_{N a}^{C u}=29, \alpha_{N a}^{Z n}=11$ e $\alpha_{N a}^{H}=22$ para os grupos sulfônicos. Os coeficientes de seletividade indicam que o $\mathrm{Cu}^{2+}$ substitui mais facilmente o $\mathrm{Na}^{+}$do que o $\mathrm{Zn}^{2+}$ e $\mathrm{H}^{+}$, para ambos os grupos funcionais. Além disso, os íons de cobre e zinco tem uma maior afinidade para os grupos carboxílicos do que para os grupos sulfônicos. 


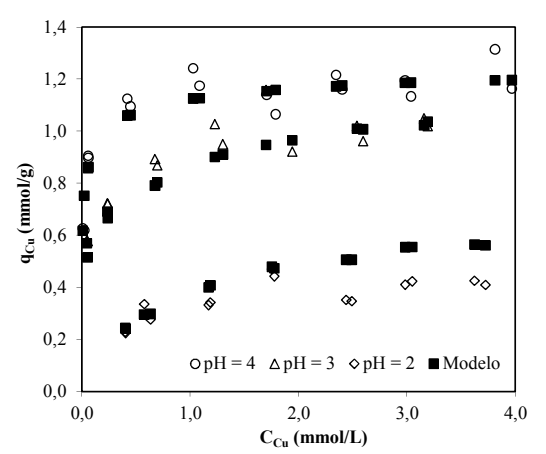

(a)

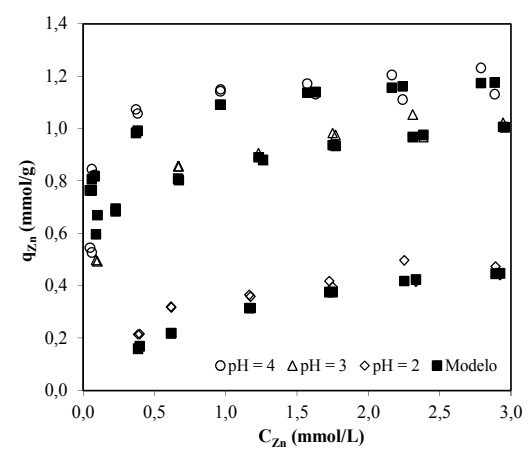

(b)

Figura 2 - Dados experimentais do equilíbrio de biossorção e modelo de troca iônica para diferentes valores de $\mathrm{pH}$ utilizando a alga-Na: cobre (a) e zinco (b).

$\mathrm{O}$ pH desempenha um papel importante na capacidade de adsorção dos metais. A biossorção de $\mathrm{Cu}^{2+} \mathrm{e} \mathrm{Zn}^{2+}$ aumenta fortemente com o aumento do $\mathrm{pH}$, uma vez que para valores de $\mathrm{pH}$ mais elevados, mais grupos funcionais ionizados estão disponíveis para ligação dos metais e a competição para os sítios ativos entre $\mathrm{H}^{+}$e $\mathrm{Cu}^{2+}$ ou $\mathrm{Zn}^{2+}$ diminui devido à menor concentração de $\mathrm{H}^{+}$. Em $\mathrm{pH}$ 2,0, os grupos carboxílicos não estão ionizados e consequentemente, não estão disponíveis para a ligação de cobre e zinco. A capacidade máxima de remoção em $\mathrm{pH} 2,0$ é de aproximadamente $1,0 \mathrm{mEq} / \mathrm{g}$ para íons de

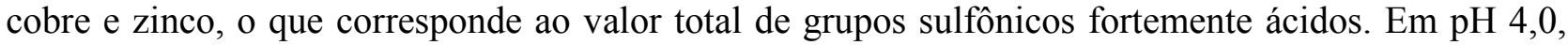
quase todos os sítios de ligação foram ocupados por íons de cobre e zinco, atingindo capacidade máxima de biossorção próximo de $2,4 \mathrm{mEq} / \mathrm{g}$, apresentando assim, um desempenho comparável aos melhores biossorventes encontrados na literatura.

\subsection{Cinética de troca catiônica com alga saturada com sódio}

As cinéticas de troca catiônica para os íons de cobre e zinco foram realizadas utilizando a alga saturada com sódio para três valores de $\mathrm{pH}$ iniciais (2,0 (dados não mostrados neste trabalho), 3,0 (dados não mostrados neste trabalho) e 4,0). Os dados experimentais das cinéticas de troca iônica em $\mathrm{pH} 4,0$ para o cobre e zinco são apresentados na Figura $3\left(\left[\mathrm{Cu}^{2+}\right]_{0}=100 \mathrm{mg} / \mathrm{L}=3,15 \mathrm{mEq} / \mathrm{L} \mathrm{e}\left[\mathrm{Zn}^{2+}\right]_{0}=100\right.$ $\mathrm{mg} / \mathrm{L}=3,06 \mathrm{mEq} / \mathrm{L}$ ). O perfil cinético de todas as espécies (prótons, sódio, cobre e zinco) envolvidas no processo também está representado e os perfis do modelo de transferência de massa.

Os íons de sódio presentes na fase sólida são trocados por cobre, zinco e prótons presentes na fase líquida, que mostra a capacidade de troca iônica natural da macroalga marinha. A adsorção é rápida, numa primeira fase, e diminui até atingir a saturação. Na primeira fase são ocupados os sítios ativos com maior afinidade para o metal e mais acessíveis fisicamente. Numa segunda fase, mais lenta, são ocupados gradualmente os sítios com menor afinidade para o íon metálico e que estão menos acessíveis. $\mathrm{O}$ processo de troca iônica atinge o equilíbrio em menos de 100 minutos, o que indica o envolvimento de forças intermoleculares fracas entre os grupos funcionais e as espécies iônicas. 


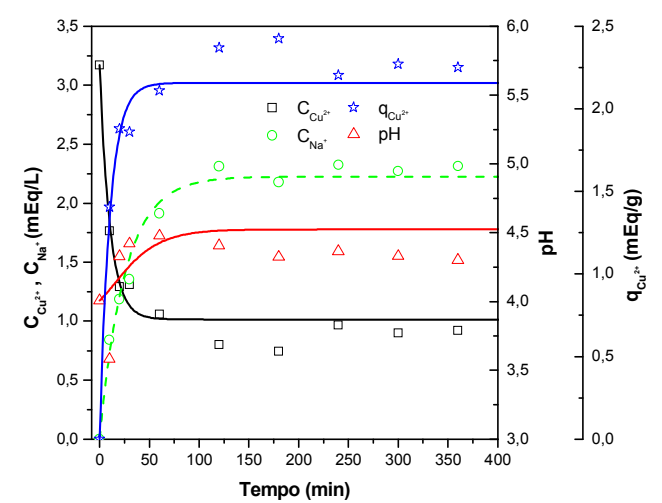

(a)

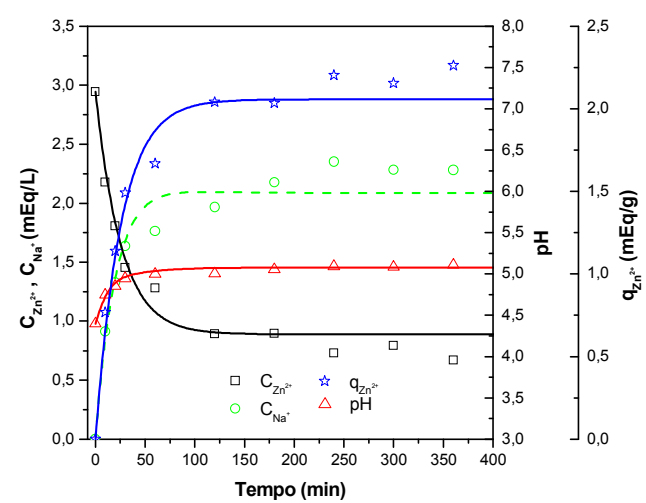

(b)

Figura 3 - Concentrações experimentais (pontos) e estimadas (linhas) de espécies iônicas na fase líquida e sólida, para a biossorção de $\mathrm{Cu}^{2+}$ (a) e $\mathrm{Zn}^{2+}$ (b) na alga-Na em pH 4,0.

Um modelo de transferência de massa reportado em nosso trabalho anterior (Hackbarth et al., 2014) foi aplicado para ajustar os dados experimentais. A Figura 3 apresenta as curvas obtidas pelo modelo de transferência de massa para os experimentos cinéticos para todas as espécies iônicas $\left(\mathrm{H}^{+}, \mathrm{Na}^{+}, \mathrm{Cd}^{2+} \mathrm{e} \mathrm{Pb}^{2+}\right)$ em fase líquida e sólida, mostrando uma boa concordância com os dados experimentais.

Os valores obtidos dos coeficientes de difusividade homogênea do íon na partícula $\left(D_{h}\right)$ para alga-Na, considerando a espessura das partículas placas plana de $0,1 \mathrm{~mm}(R=0,05 \mathrm{~mm})$, foram na faixa de $4,4 \times 10^{-9}$ e $1,2 \times 10^{-8} \mathrm{~cm}^{2} / \mathrm{s}$ para o $\mathrm{Cu}^{2+}$ e $\mathrm{Zn}^{2+}, 4,8 \times 10^{-9}$ e $7,5 \times 10^{-8} \mathrm{~cm}^{2} / \mathrm{s}$ para o $\mathrm{Na}^{+}$e $1,5 \times 10^{-}$ ${ }^{9}$ e $8,0 \times 10^{-8} \mathrm{~cm}^{2} / \mathrm{s}$ para $\mathrm{H}^{+}$. A partir dos perfis cinéticos observou-se também que os íons de hidrogênio se difundem mais rapidamente do que os íons de sódio, chumbo e cádmio, o que está de acordo com o raio do íon hidratado $\left(r_{h y b}\right)$ (Marcus; Kertes, 1969) $\left(2,82 \AA\right.$ para $\mathrm{H}^{+} ; 3,58 \AA$ para $\mathrm{Na}^{+} ; 4,19 \AA$ para $\mathrm{Cu}^{2+}$ e 4,30 para $\mathrm{Zn}^{2+}$ ) e os coeficientes de difusão molecular dados pela expressão de Nernst-Haskell $\left(D_{m}=7,2 \times 10^{-6} \mathrm{~cm}^{2} / \mathrm{s}-\mathrm{Cu}^{2+}, 6,9 \times 10^{-6} \mathrm{~cm}^{2} / \mathrm{s}-\mathrm{Zn}^{2+}, 1,3 \times 10^{-5} \mathrm{~cm}^{2} / \mathrm{s}-\mathrm{Na}^{+}, 9,3 \times 10^{-}\right.$ $\left.{ }^{5} \mathrm{~cm}^{2} / \mathrm{s}-\mathrm{H}^{+}\right)$.

\section{CONCLUSÃO}

A capacidade natural de troca catiônica da macroalga foi atribuída principalmente aos grupos funcionais ácidos (fracamente ácido $(\mathrm{COOH} ; 1,5 \mathrm{mEq} / \mathrm{g})$ e fortemente ácidos $\left(\mathrm{SO}_{3} \mathrm{H} ; 1,0 \mathrm{mEq} / \mathrm{g}\right)$ ). A liberação de metais leves durante a adsorção de $\mathrm{Cu}^{2+}$ e $\mathrm{Zn}^{2+}$ revelou que o principal mecanismo de biossorção envolvido é a troca iônica. O grau de dissociação dos grupos ácidos fracos é muito influenciado pelo $\mathrm{pH}$ da solução, afetando a capacidade de troca iônica em valores de $\mathrm{pH}$ baixos. A elevada seletividade destes biomateriais indica que eles podem ser utilizados para a recuperação de metais de grande valor presentes nos diferentes efluentes. A lei de ação das massas para a mistura ternária, $\mathrm{Cu}^{2+} / \mathrm{H}^{+} / \mathrm{Na}^{+}$ou $\mathrm{Zn}^{2+} / \mathrm{H}^{+} / \mathrm{Na}^{+}$, foi capaz de prever os dados de equilíbrio. Coeficientes de seletividade indicam que íons de $\mathrm{Cu}^{2+}$ substituem facilmente íons de $\mathrm{Na}^{+}$do que os íons de $\mathrm{Zn}^{2+} \mathrm{e} \mathrm{H}^{+}$, para ambos os grupos funcionais. Em pH 4,0, quase todos os sítios de ligação foram ocupados por íons 


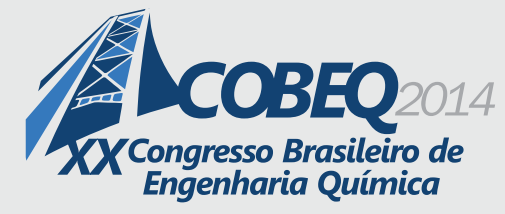

de cobre e zinco, atingindo a capacidade máxima de adsorção próxima de $2,4 \mathrm{mEq} / \mathrm{g}$. Um modelo de transferência de massa, usando a lei de ação das massas para descrever o equilíbrio ternário, e considerando o modelo da força motriz linear para descrever a resistência à difusão intrapartícula, foi capaz de prever os perfis cinéticos para ambos os sistemas ternários, $\mathrm{Cu}^{2+} / \mathrm{H}^{+} / \mathrm{Na}^{+}$ou $\mathrm{Zn}^{2+} / \mathrm{H}^{+} / \mathrm{Na}^{+}$, num sistema em batelada.

\section{REFERÊNCIAS}

DAVIS, T. A.; VOLESKY, B.; MUCCI, A. A review of the biochemistry of heavy metal biosorption by brown algae. Water Res., v. 37, n. 18, p. 4311-30, 2003.

GIRARDI, F.; HACKBARTH, F. V.; DE SOUZA, S. M. A. G. U.; DE SOUZA, A. A. U.; BOAVENTURA, R. A. R.; VILAR, V. J. P. Marine macroalgae Pelvetia canaliculata (Linnaeus) as natural cation exchanger for metal ions separation: A case study on copper and zinc ions removal. Chem. Eng. J., v. 247, n. 0, p. 320$329,2014$.

GÓMEZ-ORDÓÑEZ, E.; RUPÉREZ, P. FTIR-ATR spectroscopy as a tool for polysaccharide identification in edible brown and red seaweeds. Food Hydrocolloid., v. 25, n. 6, p. 1514-1520, 2011.

HACKBARTH, F. V.; GIRARDI, F.; DE SOUZA, S. M. A. G. U.; DE SOUZA, A. A. U.; BOAVENTURA, R. A. R.; VILAR, V. J. P. Marine macroalgae Pelvetia canaliculata (Phaeophyceae) as a natural cation exchanger for cadmium and lead ions separation in aqueous solutions. Chem. Eng. J., v. 242, n. 0, p. 294$305,2014$.

MARCUS, Y.; KERTES, A. S. Ion Exchange and Solvent Extraction of Metal Complexes, Wiley Interscience, John Wiley \& Sons, London. 1969.

MATHLOUTHI, M.; KOENIG, J. L. Vibrational spectra of carbohydrates. Adv. Carbohyd. Chem. Bi., v. 44, p. 7-89, 1987.

OLIVEIRA, R. C.; HAMMER, P.; GUIBAL, E.; TAULEMESSE, J.-M.; GARCIA JR, O. Characterization of metal-biomass interactions in the lanthanum(III) biosorption on Sargassum sp. using SEM/EDX, FTIR, and XPS: Preliminary studies. Chem. Eng. J., v. 239, n. 0, p. 381-391, 2014.

SUBBAIAH, M. V.; YUVARAJA, G.; VIJAYA, Y.; KRISHNAIAH, A. Equilibrium, kinetic and thermodynamic studies on biosorption of $\mathrm{Pb}$ (II) and $\mathrm{Cd}$ (II) from aqueous solution by fungus (Trametes versicolor) biomass. J. Taiwan Inst. Chem. Eng., v. 42, n. 6, p. 965-971, 2011.

VOLESKY, B. Detoxification of metal-bearing effluents: biosorption for the next century. Hydrometallurgy, v. 59, n. 2-3, p. 203-216, 2001. 